\title{
Pancreatic perfusion of healthy individuals and type 1 diabetic patients as assessed by magnetic resonance perfusion imaging
}

\author{
B. Hirshberg • M. Qiu • A. M. G. Cali • R. Sherwin • \\ T. Constable • R. A. Calle • M. G. Tal
}

Received: 25 March 2009/Accepted: 7 May 2009 /Published online: 3 June 2009

(C) Springer-Verlag 2009

\begin{abstract}
Aims/hypothesis Loss of pancreatic beta cell mass and function leads to the development of diabetes mellitus. Currently there is no technical way to non-invasively image islet function and mass. Murine models suggest that islets are highly vascularised organs that make a significant contribution to the total pancreatic blood flow. The current study was undertaken to test with arterial spin labelling (ASL) magnetic resonance imaging if islet mass and/or stimulation of human pancreatic islets by hyperglycaemia can differentially increase whole-pancreas perfusion, thereby distinguishing non-diabetic from type 1 diabetic patients.

Methods We assessed pancreatic blood flow using ASL at baseline, during a hyperglycaemia clamp study (glucose at $11 \mathrm{mmol} / \mathrm{l}$ ) and during recovery to euglycaemia.

Results Seventeen healthy volunteers and seven type 1 diabetic patients were studied. In healthy volunteers we observed no change in pancreatic blood flow during the three phases of the study. A trend for an increase in blood flow was observed in the two control tissues, the liver and kidney. Similarly, there was no significant difference in
\end{abstract}

Electronic supplementary material The online version of this article (doi:10.1007/s00125-009-1406-8) contains supplementary material, which is available to authorised users.

\footnotetext{
B. Hirshberg $\cdot$ R. A. Calle

Pfizer,

New London, CT, USA

M. Qiu $\cdot$ T. Constable $\cdot$ M. G. Tal $(\bowtie)$

Yale University, Diagnostic Radiology,

20 York Street,

New Haven, CT 06510, USA

e-mail: Michael.tal@yale.edu

A. M. G. Cali $\cdot$ R. Sherwin

Yale University, Endocrine Department,

New Haven, CT, USA
}

blood flow during the three stages (baseline, hyperglycaemia and recovery) in diabetic patients and there was no significant difference observed between diabetic patients and normal volunteers.

Conclusions/interpretation Our data suggest that in humans neither increased demand nor islet mass has a substantial influence on pancreatic perfusion. It is possible, however, that the current state-of-the art imaging technology employed in this study might not be sensitive enough to distinguish between a true effect and noise.

Trial registration: ClinicalTrials.gov NCT00280085

Funding: This study was fully funded by a grant from Pfizer.

Keywords Arterial spin labelling · Islet function · Islet mass · Magnetic resonance imaging · Pancreatic perfusion · Type 1 diabetes mellitus
Abbreviations
ASL Arterial spin labelling
FISP Fast imaging with steady precession
MR Magnetic resonance
MRI Magnetic resonance imaging
PASL Pulsed arterial spin labelling

\section{Introduction}

One of the underlying causes of all forms of diabetes is beta cell failure [1]. The functional capacity of islets can be assessed in vivo in humans or animals by measuring insulin secretion during an IVGTT or OGTT, an arginine stimulation test or a mixed-meal stimulation test. At present, determinations of cell mass are done on excised pancreases. 
Data from cadaveric pancreases suggest that patients with long-standing type 2 diabetes lose up to $50 \%$ of the beta cell mass [2].

Quantifying pancreatic beta cells would allow us to: (1) differentiate preservation of insulin-secretory capacity related to functional preservation vs that related to beta cell mass preservation or enhancement; (2) provide a clinical examination to monitor beta cell number, mass and function; and (3) identify at-risk individuals to be monitored prior to onset of diabetes.

Although significant progress has been made in imaging anatomical, physiological, biological and genetic markers in other organs and disease states, there is a paucity of imaging research currently addressing the endocrine pancreas. In the last few years we have seen a technological leap in non-invasive imaging technologies such as magnetic resonance (MR) imaging (MRI). Thus, there is an exciting opportunity to apply the new imaging techniques to the pancreatic beta cell [3-7].

The islets of Langerhans are highly vascularised with fenestrated capillaries. These islets make up only $1-2 \%$ of the pancreatic mass, but receive $10-30 \%$ of the pancreatic blood flow [8]. Islet blood flow is highly regulated in response to changes in function (such as response to elevated glucose) and has been measured in experimental animals to be as high as $6 \mathrm{ml}$ (g tissue $)^{-1} \mathrm{~min}^{-1}[8]$. Transplantation of islets encourages growth of these specialised capillaries in the region of engraftment [9]. Functional imaging techniques that assess blood volume, blood flow, vascular permeability and oxygenation hold promise for visualising the islet and defining its physiology [10].

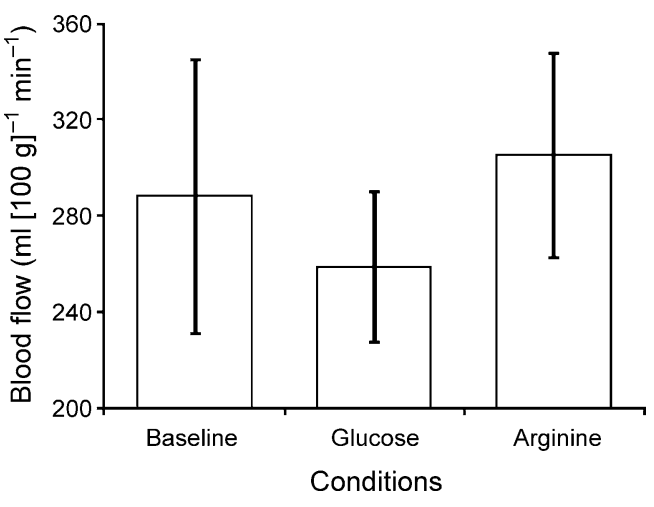

Fig. 2 Pancreatic tissue blood flow at baseline and during hyperglycaemia (glucose) and arginine stimulation in seven healthy individuals. Values are means $\pm \mathrm{SD}$

We hypothesised that an increase in metabolic demand, such as occurs during hyperglycaemia, would increase whole-pancreas perfusion and that the pancreatic perfusion at baseline and in response to hyperglycaemia would be substantially different between healthy volunteers (individuals with preserved beta cell function) vs patients with type 1 diabetes (lack of beta cell mass and function). The aim of the current study was to test this hypothesis by means of MRI, specifically arterial spin labelling (ASL) [6].

\section{Methods}

Participants The healthy non-diabetic group consisted of 17 individuals (ten men and seven women) between the ages of 19 and 59 years (mean 38 years) who had no

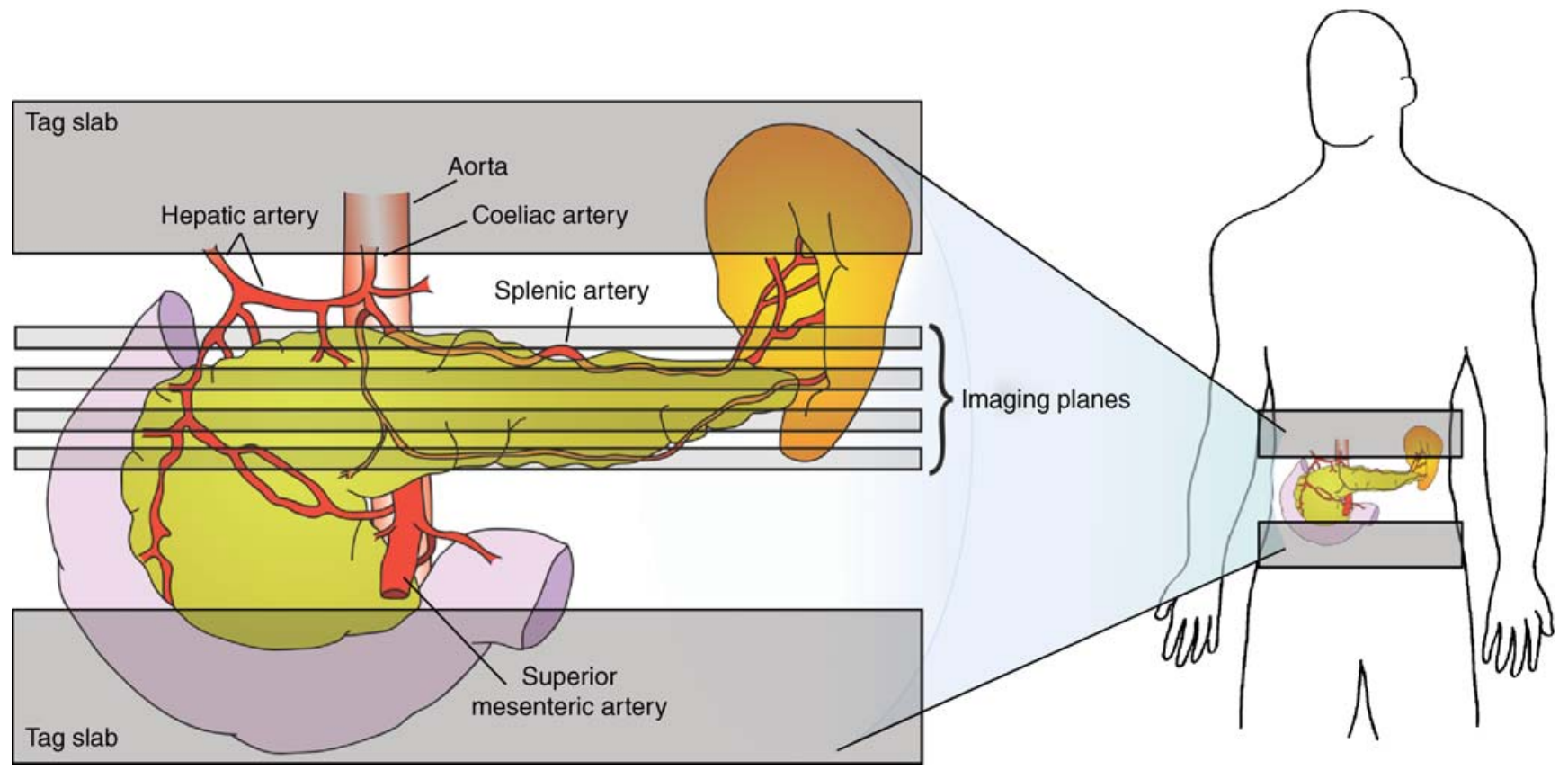

Fig. 1 Regions of the pancreatic tissue were identified manually for each patient 
Fig. 3 Perfusion of the pancreas, liver and kidney in 17 healthy volunteers (a) and seven type 1 diabetic patients $(\mathbf{b})$ at baseline (white bars), during a hyperglycaemic clamp (grey bars) and during the recovery stage (black bars). Values are means \pm SD
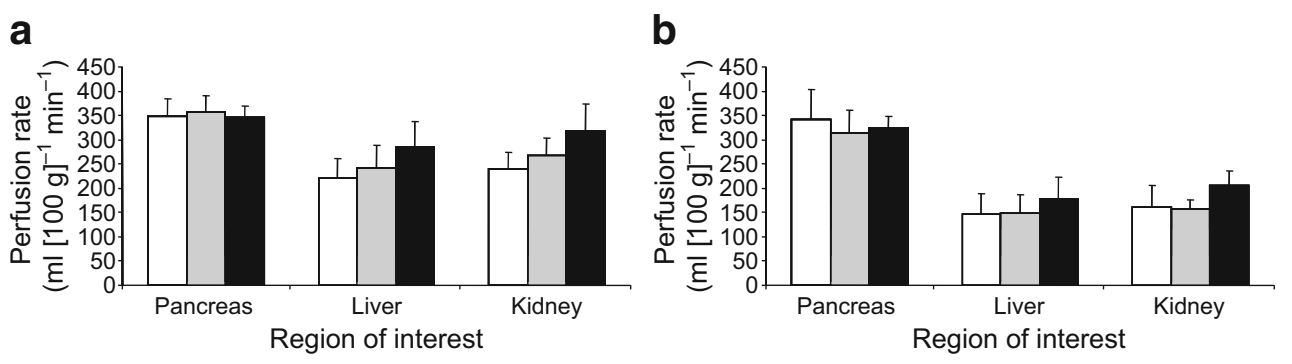

clinically relevant abnormalities identified by medical history, physical examination, ECG and clinical laboratory tests. Their BMIs were $18-30 \mathrm{~kg} / \mathrm{m}^{2}$ (mean $24.7 \mathrm{~kg} / \mathrm{m}^{2}$ ) and total body weights $>50 \mathrm{~kg}$. Seven type 1 diabetic patients (one man and six women), 21-51 years old (mean 35 years) were selected based on the American Diabetes Association definition [11]. Additional inclusion criteria were: fasting plasma $\mathrm{C}$-peptide $<0.06 \mathrm{nmol} / 1$ and no change in their diabetic treatment regimen for at least 2 months prior to the study. All were being treated with either three or four insulin injections per day or an insulin pump. The average BMI of the diabetic patients was $26.2 \mathrm{~kg} / \mathrm{m}^{2}$ with a mean $\mathrm{HbA}_{1 \mathrm{c}}$ of $7.1 \%$ (range $6.6-8.3 \%$ ). The Yale University School of Medicine Human Investigation Committee approved this study and all participants provided informed, written consent before participation in the study.

Hyperglycaemic clamp protocol Following a basal period, plasma glucose levels were acutely raised to a range of 8.8$11.1 \mathrm{mmol} / \mathrm{l}$ by infusing a priming dose of $20 \%$ (wt/vol.) dextrose solution in a decreasing logarithmic manner. Subsequently, plasma glucose was held constant at this new hyperglycaemic level for $30 \mathrm{~min}$ by adjustment of the $20 \%$ dextrose infusion. Thereafter the glucose infusion was discontinued. In those participating in the arginine substudy, 20 min after raising the glucose levels to the range of $8.8-11.1 \mathrm{mmol} / \mathrm{l}$ an infusion of $5 \mathrm{~g}$ arginine over a $1 \mathrm{~min}$ period (as a $10 \% \mathrm{wt} / \mathrm{vol}$. solution) was given.

Imaging protocols Imaging was performed on a $1.5 \mathrm{~T}$ whole-body scanner (Sonata; Siemens Medical Systems, Erlangen, Germany). The built-in body coil was used for transmission and a six-element surface coil for signal reception. MR perfusion-weighted images were acquired using a pulsed ASL (PASL) sequence. It was modified from a fast imaging protocol with a steady procession FISP sequence [12] by incorporating the proximal inversion with a control for off-resonance effects spin labelling technique [6]. Full description of the PASL protocol is presented in the Electronic supplementary material (ESM).

Data processing Regions of the pancreatic tissue were identified manually for each patient (Fig. 1). Absolute pancreatic blood flow was estimated on a per voxel basis on the perfusion data collected at each time point, and the mean blood flow was then calculated within the pancreatic region. By doing this we obtained a time course representing the pancreatic blood measured at different times in a patient. The temporal mean and SD were then estimated for each of the three conditions: euglycaemic, hyperglycaemic and recovery.

\section{Results}

We first wished to test if the addition of arginine (on top of a hyperglycaemic clamp) would enhance blood flow to the pancreas. Seven healthy individuals were tested under these conditions. As can be seen in Fig. 2, we did not observe a significant difference in pancreatic blood flow following the arginine induction, and thus adding the use of arginine to the protocol was discontinued.

Next we assessed three organs, the pancreas, liver and kidney (the last two as controls) during three stages: baseline, hyperglycaemia and recovery (Fig. 3a). We
Fig. 4 Glucose (a) and Cpeptide (b) levels during the hyperglycaemic clamp in 17 healthy volunteers (squares, glucose+arginine; diamonds, glucose only) and seven type 1 diabetic patients (triangles) a

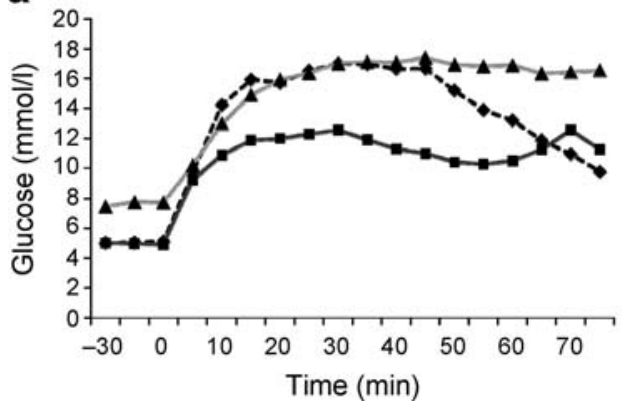

b

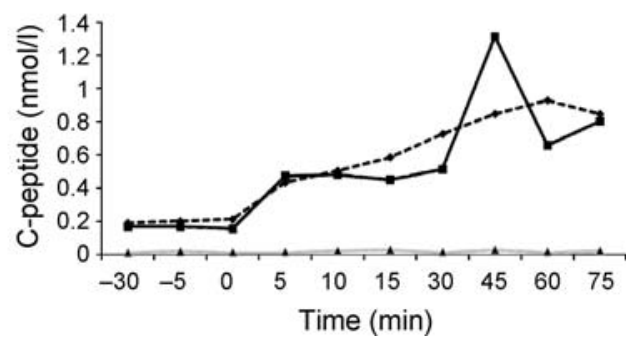


observed no change in pancreatic blood flow during the three stages. A trend for an increase in blood flow was observed in the two control tissues, the liver and kidney.

We finally assessed pancreatic blood flow in seven type 1 diabetic patients (Fig. 3b). We did not observe differences in blood flow during the three stages (baseline, hyperglycaemia and recovery). These findings were identical to the observations in normal individuals.

The hyperglycaemic clamp resulted in the expected increase in glucose and C-peptide levels (Fig. 4a, b).

\section{Discussion}

We here report our attempt to assess functional islet mass using pancreatic perfusion as an imaging biomarker. Our data suggest that neither islet mass nor hyperglycaemia has an effect on pancreatic perfusion, as assessed with MRI ASL. The technique used, state-of-the art ASL, failed to distinguish normal volunteers with intact beta cell function and mass from patients with long-standing type 1 diabetes, representing a population lacking most beta cell function and mass. Furthermore, even physiological conditions representing maximal beta cell stimulation (high glucose levels during a hyperglycaemic clamp in conjunction with arginine) have not shown significant flow changes. This contrasted with our control organs (the kidney and liver) where differences were seen, demonstrating higher perfusion during the hyperglycaemia phase.

The failure to distinguish normal volunteers and type 1 diabetic patients may result from either a biological or technical cause or a combination of both.

The biological causes are as follows: (1) Changes in pancreatic islet blood flow have been demonstrated mostly in murine models of diabetes. The assessment was done by an injection of marked microspheres with rapid (10 s) killing of the animals following injection [13]. Thus the results described in those studies may not represent dynamic change in flow as assessed in the current study. (2) Human islet physiology differs from murine physiology. Thus, we cannot exclude that human islet perfusion is not affected to the same degree as murine models by changes in glucose levels or other stimulating factors. (3) Even if human islet perfusion does change in response to stimuli, it is possible that these changes have little effect on wholepancreas perfusion.

The technical reasons are as follows: (1) Islets make up only $1-2 \%$ of the pancreatic mass and are distributed unevenly throughout the pancreas and may be stimulated in various degrees during hyperglycaemia. The noise-to-signal ratio may be too great to give a meaningful result. (2) The pancreas is located below the liver, close to the stomach, and behind the intestines. Small irregular movements of the surrounding organs may cause image intensity changes during MRI. Since ASL is geared to detect small differences induced by labelled arterial water spins, the artefacts induced by the surrounding organ movements may reduce the ability of this technique to assess pancreatic blood flow. (3) The pancreas is a relatively small organ so there may be an insufficient number of voxels to allow a more robust MR signal. In addition, respiratory movements may also prevent accurate measurement of perfusion.

In conclusion, this is the first attempt to image native human islet function using perfusion imaging. No differences were seen between normal volunteers and patients with type 1 diabetes, neither prior to nor after clamping glucose levels using a hyperglycaemic clamp. This suggests that islet perfusion has little effect on whole-pancreas perfusion. We cannot exclude, however, that the current state-of-the art imaging technique employed in this study is not sensitive enough to distinguish between a true effect and noise.

Acknowledgements The authors would like to take this opportunity to thank K. Scheffler (University of Basel) for his generosity in providing the True FISP PASL sequence used in this research. We would like to thank E. Hintz, A. O'Connor, M. Smolgovsky and H. Sarofin for help in executing these studies, and G. Mancini (Yale University, Diagnostic Radiology) for her illustrations and art work.

Duality of interest The authors declare that there is no duality of interest associated with this manuscript.

\section{References}

1. Bergman RN, Ader M, Huecking K, van Citters G (2002) Accurate assessment of beta-cell function: the hyperbolic correction. Diabetes 51:S212-S220

2. Butler AE, Janson J, Bonner-Weir S, Ritzel R, Rizza RA, Butler PC (2003) Beta-cell deficit and increased beta-cell apoptosis in humans with type 2 diabetes. Diabetes 52:102-110

3. Buxton RB, Frank LR, Wong EC, Siewert B, Warach S, Edelman RR (1998) A general kinetic model for quantitative perfusion imaging with arterial spin labeling. Magn Reson Med 40:383-396

4. Detre JA, Leigh JS, Williams DS, Koretsky AP (1992) Perfusion imaging. Magn Reson Med 23:37-45

5. Luh WM, Wong EC, Bandettini PA, Hyde JS (1999) QUIPSS II with thin-slice TI1 periodic saturation: a method for improving accuracy of quantitative perfusion imaging using pulsed arterial spin labeling. Magn Reson Med 41:1246-1254

6. Wong EC, Buxton RB, Frank LR (1998) Quantitative imaging of perfusion using a single subtraction (QUIPSS and QUIPSS II). Magn Reson Med 39:702-708

7. Noworolski SM, Henry RG, Vigneron DB, Kurhanewicz J (2005) Dynamic contrast-enhanced MRI in normal and abnormal prostate tissues as defined by biopsy, MRI, and 3D MRSI. Magn Reson Med 53:249-255

8. Jansson L (1994) The regulation of pancreatic islet blood flow. Diabetes Metab Rev 10:407-416

9. Hirshberg B, Mog S, Patterson N, Leconte J, Harlan DM (2002) Histopathological study of intrahepatic islets transplanted in the nonhuman primate model using Edmonton protocol immunosuppression. J Clin Endocrinol Metab 87:5424-5429 
10. Paty BW, Bonner-Weir S, Laughlin MR, McEwan AJ, Shapiro AM (2004) Toward development of imaging modalities for islets after transplantation: insights from the National Institutes of Health Workshop on Beta Cell Imaging. Transplantation 77:1133-1137

11. American Diabetes Association (2005) Diagnosis and classification of diabetes mellitus. Diabetes Care 28:S37-S42
12. Scheffler K, Seifritz E, Bilecen D et al (2001) Detection of BOLD changes by means of a frequency-sensitive trueFISP technique: preliminary results. NMR Biomed 14:490-496

13. Carlsson PO, Olsson R, Kallskog O, Bodin B, Andersson A, Jansson L (2002) Glucose-induced islet blood flow increase in rats: interaction between nervous and metabolic mediators. Am J Physiol Endocrinol Metab 283:E457-E464 